\title{
A Symplectic Structure for Connections on Surfaces with Boundary
}

\author{
Christopher King ${ }^{1}$, Ambar Sengupta ${ }^{2}$ \\ ${ }^{1}$ Department of Mathematics, Northeastern University, Boston, MA 02115, USA \\ 2 Department of Mathematics, Louisiana State University, Baton Rouge, Louisiana 70803-4918, \\ USA
}

Received: 11 November 1994/in revised form: 24 March 1995

\begin{abstract}
For compact surfaces with one boundary component, and semisimple gauge groups, we construct a closed gauge invariant 2-form on the space of flat connections whose boundary holonomy lies in a fixed conjugacy class. This form descends to the moduli space under the action of the full gauge group, and provides an explicit description of a symplectic structure for this moduli space.
\end{abstract}

\section{Introduction}

We construct a natural gauge invariant 2-form on the space of all connections over a compact surface with one boundary component, for semisimple gauge groups. When restricted to the space of flat connections whose boundary holonomy lies in a fixed conjugacy class, this 2-form is closed and descends to the moduli space under the action of the full gauge group. We show that for a non-empty open set of conjugacy classes, this gives a symplectic structure on the moduli space.

The moduli space of flat connections on a closed surface has been studied from many viewpoints ([AB, BG, Go, Hi, Hu, HJ, J, JW 1,2, Ka, KS1,2, Se2, We, Wi1,2]). A symplectic structure on the moduli space of flat connections with fixed boundary holonomy conjugacy class has been discussed in [We] and is also mentioned in [A]. Several works, including [A, BG, J, Wil], have investigated the closely related issue of a symplectic structure on the moduli space of flat connections on a surface with marked points.

The present paper uses results from [KS3], where a 2-form was constructed on the moduli space of flat connections on a compact surface with boundary, under the group of based gauge transformations. Ideas and techniques from [KS1] and [KS2] are also used. An underlying theme is the study of certain naturally defined differential forms on the moduli space of flat connections and their counterparts on the infinite dimensional space of all connections.

The paper is organised as follows. In Sect. 2 the general setup is described and the infinite dimensional space $\mathcal{A}$ of all connections is introduced, along with certain (infinite dimensional) subsets $\mathcal{A}^{f l}, \mathcal{A}(\Theta)$ and $\mathcal{A}^{f l}(\Theta)$, of interest. In general we use $\Theta$ to denote a conjugacy class in the group $G$, and so we attach the symbol $\Theta$ 
to an object (a space or a form) which depends on a choice of conjugacy class for the boundary holonomy. The various finite dimensional moduli spaces $\mathcal{M}_{o}, \mathcal{M}_{o}(\Theta)$, $\mathcal{M}$ and $\mathcal{M}(\Theta)$ are also defined here. The subscript " $o$ " indicates that these moduli spaces are obtained under the action of the based gauge group, consisting of gauge transformations which are the identity at a fixed point $o$ on the surface. We close Sect. 2 by recalling the definition of the Atiyah-Bott symplectic form on $\mathcal{A}$ (which we denote by $\Omega$ ).

In Sect. 3 we establish smoothness of the moduli spaces for generic conjugacy classes. We then introduce several modifications of the Atiyah-Bott symplectic form, leading to a closed 2-form $\bar{\Omega}_{\Theta}$ on the moduli space of interest, $\mathcal{M}(\Theta)$ (where appropriate we place a bar over a form on a quotient space to indicate that it descends from the corresponding unbarred form on the covering space). In Sect. 4 we establish non-degeneracy of $\bar{\Omega}_{\Theta}$ for a non-empty open subset of conjugacy classes in $G$. Finally, in Sect. 5 we identify a 2 -form $\Omega_{\Theta}$ on the infinite dimensional space $\mathcal{A}$ which descends to the 2 -form $\bar{\Omega}_{\Theta}$ on the quotient $\mathcal{M}(\Theta)$, and discuss its properties.

\section{Notation and Background}

2.1. The surface $\Sigma$, and the loops $A_{1}, B_{1}, \ldots, A_{g}, B_{g}, C$ based at $o \in \Sigma$. We will work with a compact oriented two-dimensional manifold $\Sigma$, of genus $g \geqq 1$, which has connected boundary $\partial \Sigma$. We shall also use piecewise smooth loops $A_{1}, B_{1}, \ldots, A_{g}, B_{g}, C:[0,1] \rightarrow \Sigma$, all based at a point $o \in \Sigma$, which generate the fundamental group $\pi_{1}(\Sigma, o)$, subject to the relation that $C \bar{B}_{g} \bar{A}_{g} B_{g} A_{g} \cdots \bar{B}_{1} \bar{A}_{1} B_{1} A_{1}$ is homotopic to the constant loop at $o$, wherein we denote by $\bar{X}$ the reverse of any path $X$. The loop $C$ is of the form $\bar{L} C^{\prime} L$, where $C^{\prime}$ is a simple loop around the boundary $\partial \Sigma$ and $L$ is a path from $o$ to the initial point of $C^{\prime}$; the loops $A_{i}$ and $B_{l}$ are also of the form $A_{i}=\bar{L} A_{i}^{\prime} L$ and $B_{i}=\bar{L} B_{i}^{\prime} L$. A detailed description of these loops is given in [KS3: Sect. 2.1], but we shall not need such details here.

2.2. The group $G$, Lie algebra $g$, and metric $\langle\cdot, \cdot\rangle$. Let $G$ be a compact connected semisimple Lie group. The Lie algebra of $G$ will be denoted $g$, and we will use an Ad-invariant metric $\langle\cdot, \cdot\rangle$ on $\underline{g}$.

2.3. Conjugacy classes $\Theta$. We shall denote by $\Theta$ a typical conjugacy class in $G$; i.e. $\Theta=\left\{x c x^{-1}: x \in G\right\}$ for some $c \in G$. Let $Z(c)$ be the closed subgroup $\{x \in$ $\left.G: x c x^{-1}=c\right\}$. The map $G \rightarrow G: x \mapsto x c x^{-1}$ induces an immersion $G / Z(c) \rightarrow G$ : $x . Z(c) \mapsto x c x^{-1}$, which maps homeomorphically onto $\Theta$. Thus $\Theta$ is a submanifold of $G$, and the map $G / Z(c) \rightarrow \Theta: x \cdot Z(c) \mapsto x c x^{-1}$ is a diffeomorphism.

2.4. The spaces $\mathcal{A}$ and $\mathcal{A}^{f l}$; the bundle $P$. Let $\pi: P \rightarrow \Sigma$ be a principal $G$-bundle over $\Sigma$, and $\mathcal{A}$ the affine space of all connections on $P$. A connection $\omega \in \mathcal{A}$ is flat if $d \omega+\frac{1}{2}[\omega, \omega]=0$. We denote by $\mathcal{A}^{f l}$ the space of all flat connections in $\mathcal{A}$. The bundle $P$ is trivial, and, by fixing a smooth section $\sigma: \Sigma \rightarrow P$, and identifying $\omega \in \mathcal{A}$ with the $g$-valued 1 -form $\sigma^{*} \omega$ on $\Sigma$, we can identify $\mathcal{A}$ with the space of all $g$-valued 1 -forms on $\Sigma$. With this identification, $\mathcal{A}$ is actually a vector space, and thus a tangent vector to $\mathcal{A}$ is simply an element of $\mathcal{A}$; but we shall often use the phrase 'tangent vector to $\mathcal{A}$ ' instead of 'element of $\mathcal{A}$ ' to keep the conceptual distinction clear. 
2.5. Holonomy $h(\kappa ; \omega)$; the spaces $\mathcal{A}(\Theta)$ and $\mathcal{A}_{o}(\Theta)$. We shall denote by $h(\kappa ; \omega)$ the holonomy of a connection $\omega$ around a loop $\kappa$ on $\Sigma$ based at $o$, taking $\sigma(o)$ as the initial point. That is, $h(\kappa ; \omega)=h(1)$ if $\kappa:[0,1] \rightarrow \Sigma$ and $[0,1] \rightarrow G: t \mapsto h(t)$ solves the equation $h^{\prime}(t) \cdot h(t)^{-1}+\omega\left(\kappa^{\prime}(t)\right)=0$ subject to the initial condition $h(0)=e$ (and, in this differential equation we are viewing $\omega$ as a 1 -form on $\Sigma$ as explained in Sect. 2.4). The path $t \mapsto h(t)$ describes parallel transport by $\omega$ along $\kappa$. Among the main objects of interest to us will be the space $\mathcal{A}(\Theta)=\{\omega \in \mathcal{A}$ : $h(C ; \omega) \in \Theta\}$, where $\Theta$ is any conjugacy class in $G$. In particular we shall be concerned with the subset $\mathcal{A}^{f l}(\Theta)=\mathcal{A}^{f l} \cap \mathcal{A}(\Theta)$.

2.6. The groups $\mathcal{G}$ and $\mathcal{G}_{0}$ of gauge transformations. Let $\mathcal{G}$ be the set of all gauge transformations, i.e. $G$-equivariant diffeomorphisms $\phi: P \rightarrow P$ for which $\pi \circ \phi=\pi$. This is a group under composition. We shall also use the subgroup $\mathcal{G}_{o}=\{\phi \in \mathcal{G}$ : $\phi(u)=u\}$, where $u$ is any point on $\pi^{-1}(o)$. Identifying $\phi \in \mathcal{G}$ with the function $\hat{\phi}: \Sigma \rightarrow G$ specified by $\phi(\sigma(x))=\sigma(x) \hat{\phi}(x)$ for every $x \in \Sigma$, the group $\mathcal{G}$ may be identified with the group (under pointwise multiplication) of all smooth functions $\Sigma \rightarrow G$; then $\mathcal{G}_{o}$ consists of those functions which take the value $e$ (identity in $G$ ) at $o$.

2.7. Action of $\mathcal{G}$ on $\mathcal{A}$; the moduli spaces $\mathcal{M}_{o}, \mathcal{M}_{o}(\Theta)$, and $\mathcal{M}, \mathcal{M}(\Theta)$. The group $\mathcal{G}$ acts on $\mathcal{A}$ by pulling back connections: $\mathcal{G} \times \mathcal{A} \rightarrow \mathcal{A}:(\phi, \omega) \mapsto \phi^{*} \omega$; equivalently, $(\phi, \omega) \mapsto \operatorname{Ad}\left(\phi^{-1}\right) \omega+\phi^{-1} d \phi$, in terms of functions and $1-$ forms on $\Sigma$. It is readily verified that for any conjugacy class $\Theta$, the action of $\mathcal{G}$ carries $\mathcal{A}(\Theta)$ into itself, and also $\mathcal{A}^{f l}$ into itself. Thus we obtain the moduli spaces $\mathcal{A}^{f l} / \mathcal{G}$, $\mathcal{A}^{f l}(\Theta) / \mathcal{G}_{o}$, and $\mathcal{A}^{f l}(\Theta) / \mathcal{G}$. Holonomies around the loops $A_{1}, B_{1}, \ldots, A_{g}, B_{g}, C$ form the map

$$
\mathcal{A}^{f l} \rightarrow G^{2 g} \times G: \omega \mapsto\left(h\left(A_{1} ; \omega\right), h\left(B_{1} ; \omega\right), \ldots, h\left(A_{g} ; \omega\right), h\left(B_{g} ; \omega\right), h(C ; \omega)\right),
$$

which induces identifications

$$
\begin{array}{r}
\mathcal{A}^{f l} / \mathcal{G}_{o} \simeq \mathcal{M}_{o}=\left\{\left(a_{1}, b_{1}, \ldots, a_{g}, b_{g}, c\right)\right. \\
\left.\in G^{2 g} \times G: c b_{g}^{-1} a_{g}^{-1} b_{g} a_{g} \ldots b_{1}^{-1} a_{1}^{-1} b_{1} a_{1}=e\right\}, \\
\mathcal{A}^{f l}(\Theta) / \mathcal{G}_{o} \simeq \mathcal{M}_{o}(\Theta)=\left\{\left(a_{1}, b_{1}, \ldots, a_{g}, b_{g}, c\right) \in \mathcal{M}_{0}: c \in \Theta\right), \\
\mathcal{A}^{f l} / \mathcal{G} \simeq \mathcal{M}=\mathcal{M}_{o} / G, \quad \text { and } \quad \mathcal{A}^{f l}(\Theta) / \mathcal{G} \simeq \mathcal{M}(\Theta)=\mathcal{M}_{o}(\Theta) / G,
\end{array}
$$

where, in $(2.1 \mathrm{c})$, the quotients by $G$ are obtained from the conjugation action of $G$ on $G^{2 g} \times G$.

2.8. The symplectic structure $\Omega$ on $\mathcal{A}$. The infinite dimensional space $\mathcal{A}$ has a natural symplectic structure, which we now describe (from $[\mathrm{AB}]$ ). A tangent vector to $\mathcal{A}$ may be identified with a $\underline{g}$-valued 1 -form on $\Sigma$. Using the inner product on $\underline{g}$, we obtain from two $\underline{g}$-valued 1 -forms $\zeta$ and $\eta$ on $\Sigma$ an ordinary 2-form $\langle\zeta \wedge \eta\rangle$ on $\Sigma$ as follows:

$$
\langle\zeta \wedge \eta\rangle(X, Y)=\langle\zeta(X), \eta(Y)\rangle-\langle\zeta(Y), \eta(X)\rangle
$$


The 2-form $\Omega$ is specified as follows. If $A$ and $B$ are $g$-valued 1 -forms on $\Sigma$ representing tangent vectors in $\mathcal{A}$ then

$$
\Omega(A, B)=\int_{\Sigma}\langle A \wedge B\rangle .
$$

It is clear that $\Omega$ is invariant under the group $\mathcal{G}$ of gauge transformations, and that, being constant (independent of $\omega \in \mathcal{A}$ ), it is closed. In fact $\Omega$ is a symplectic form on $\mathcal{A}$.

\section{The 2-Form $\bar{\Omega}_{\Theta}$ on $\mathcal{M}(\Theta)$}

In this section we shall construct a 2 -form $\bar{\Omega}_{\Theta}$ on the moduli space $\mathcal{M}(\Theta)$ by modifying $\Omega$. The 2 -form $\Omega$ itself does not descend to the quotient $\mathcal{A}^{\text {fl }} / \mathcal{G}$. In [KS3], a term was added to $\Omega$ to obtain a 2 -form $\Omega_{1}$ (in [KS3] it was called $\Omega_{\text {new }}$ ). This 2 -form $\Omega_{1}$ descends as a 2 -form $\bar{\Omega}_{1}$ on $\mathcal{A}^{f l} / \mathcal{G}_{o}$ but not to $\mathcal{A}^{f l} / \mathcal{G}$, and is not closed. We shall here introduce a modification of $\Omega_{1}$ which is closed and which descends to the moduli space $\mathcal{A}^{f^{\prime} l}(\Theta) / \mathcal{G}$.

3.1. The 2-forms $\Omega_{e x}$ and $\Omega_{1}$. We use from [KS3. Def. 3.1] the 2-form $\Omega_{e x}$ on $\mathcal{A}$ defined in the following way. Recall, from Sect. 2.1, the loop $C$, part of which goes around $\partial \Sigma$. Let $A$ be a tangent vector to $\mathcal{A}$ (i.e. $A$ is a $g$-valued 1 -form on $\Sigma)$, and define

$$
\alpha:[0,1] \rightarrow \underline{g}: t \mapsto \alpha(t)=-\int_{0}^{t} \operatorname{Ad}\left(h_{s}^{-1}\right) A\left(C^{\prime}(s)\right) d s,
$$

where $s \mapsto h_{s}$ describes parallel transport along $C$. Thus $\alpha(t)$ is the variation in $h_{t}$ corresponding to the variation $A$ in the connection $\omega$ (see Eq. (5.2) in this context). Define $\beta:[0,1] \rightarrow G$ similarly with respect to a tangent vector $B$ to $\mathcal{A}$. Then :

$$
\Omega_{e x}(A, B)=-\frac{1}{2} \int_{0}^{1} \int_{0}^{1} \varepsilon_{s t}\left\langle\alpha^{\prime}(s), \beta^{\prime}(t)\right\rangle d s d t,
$$

where

$$
\varepsilon_{s t}= \begin{cases}1 & \text { if } s \leqq t \\ -1 & \text { if } s>t\end{cases}
$$

The 2-form

$$
\Omega_{1}=\Omega+\Omega_{e x}
$$

was introduced in [KS3, Def. 3.2] (denoted there by $\Omega_{\text {new }}$ ), and it was shown ([KS3, Proposition 3.3]) that it descends to a 2 -form $\bar{\Omega}_{1}$ on $\mathcal{M}_{o} \simeq \mathcal{A}^{f l} / \mathcal{G}_{o}$.

3.2. Smoothness of $\mathcal{M}_{o}(\Theta)$, and $\mathcal{M}(\Theta)$. Consider the map

$$
\Pi: G^{2 g+1} \rightarrow G:\left(a_{1}, b_{1}, \ldots, a_{g}, b_{g}, c\right) \mapsto c K,
$$

where

$$
K=b_{g}^{-1} a_{g}^{-1} b_{g} a_{g} \ldots b_{1}^{-1} a_{1}^{-1} b_{1} a_{1} .
$$

We will often view $K$ as a mapping $G^{2 g} \rightarrow G$. Thus

$$
\mathcal{M}_{o}=\Pi^{-1}(e)
$$


and

$$
\mathcal{M}_{o}(\Theta)=\mathcal{M}_{o} \cap\left(G^{2 g} \times \Theta\right)=\left(\Pi \mid G^{2 g} \times \Theta\right)^{-1}(e) .
$$

Let $\left(a_{1} A_{1}, \ldots, b_{g} B_{g}, c C\right)$ be tangent, at $\alpha=(h, c)=\left(a_{1}, b_{1}, \ldots, a_{g}, b_{g}, c\right) \in \mathcal{M}_{o}(\Theta)$, to a smooth path in $G^{2 g+1}$ lying on $\Pi^{-1}(e)$. It will be convenient to work with

$$
\left(\alpha_{1}, \ldots, \alpha_{4 g+1}\right)=\left(a_{1}, b_{1}, a_{1}^{-1}, b_{1}^{-1}, \ldots, a_{g}, b_{g}, a_{g}^{-1}, b_{g}^{-1}, c\right) \in G^{4 g} \times G ;
$$

then

$$
\alpha=\left(\left(\alpha_{j}\right)_{j \in J}, c\right)
$$

where

$$
J=\{1,2,5,6, \ldots, 4 g-3,4 g-2\} .
$$

With this notation, we can compute:

$$
\Pi(\alpha)^{-1} d \Pi_{\gamma}\left(a_{1} A_{1}, \ldots, b_{g} B_{g}, c C\right)=\operatorname{Ad}\left(K^{-1}\right) C+K^{-1} d K_{h}\left(a_{1} A_{1}, \ldots, b_{g} B_{g}\right) .
$$

The derivative of $K$ is easily computed as:

$$
K^{-1} d K_{h}\left(a_{1} A_{1}, \ldots, b_{g} B_{g}\right)=\sum_{j \in J}\left[f_{j-1}^{-1}-f_{j+2}^{-1}\right] H_{j},
$$

where

$$
f_{l}=\operatorname{Ad}\left(\alpha_{l} \cdots \alpha_{1}\right),
$$

and the vectors $H_{i}$ are specified by

$$
A_{i}=H_{4 l-3} \quad \text { and } \quad B_{i}=H_{4 i-2},
$$

and, for use in Sect. 3.3 below, we also set $H_{j+2}=-\operatorname{Ad}\left(\alpha_{j}\right) H_{J}$ for $j \in J$.

We shall now show that for $\Theta$ through a generic $c, \mathcal{M}_{o}(\Theta)$ is a smooth manifold. By Sard's theorem, the regular values of $K$ form an open dense subset $D$ of $G$. Since $c \mapsto c^{-1}$ and $c \mapsto x c x^{-1}$, for any fixed $x$, are diffeomorphisms of $G$, it follows that $D=D^{-1}$ and that the conjugation action of $G$ maps $D$ onto itself. The map $K$ is surjective (this uses semisimplicity of $G$; see [Se1, Proposition 3.6], for instance) and thus each $K^{-1}(c) \subset G^{2 g}$ is non-empty. View $\Pi$ as a map $G^{2 g} \times \Theta \rightarrow G$, where $\Theta$ is the conjugacy class of $c$. Then Eq. (3.5) implies that, for any $\alpha=(h, c) \in G^{2 g} \times G$, ker $d\left(\Pi \mid G^{2 g} \times \Theta\right)_{\alpha}^{*} \subset \operatorname{ker} d K_{h}^{*}$. Here, and always, we are taking $(d K)^{*}$ and $(d \Pi)^{*}$ to be maps between $\underline{g}$ and $\underline{g}^{2 g}$, by means of appropriate left-translations. Choosing $c \in D$ and any $\alpha=(h, c) \in \bar{\Pi}^{-1}(e)$, it follows that $\Pi \mid\left(G^{2 g} \times \Theta\right)$ is not critical at $\alpha$. Thus $\Pi \mid\left(G^{2 g} \times \Theta\right)$ is a submersion in a neighborhood of $\left(\Pi \mid G^{2 g} \times \Theta\right)^{-1}(e)$, when $\Theta$ is the conjugacy class through any point in the dense open set $D \subset G$. Consequently, for such $\Theta$, the level surface $\left(I I \mid G^{2 g} \times \Theta\right)^{-1}(e)$ is a smooth submanifold of $G^{2 g} \times \Theta$. More generally, for any $\Theta$, if the genus $g$ of the surface $\Sigma$ is at least 2 then it may be shown (as in Proposition III.B of [KS1]) that $\mathcal{M}_{o}(\Theta)$ has a non-empty open subset, its 'smooth part,' consisting of points where $\Pi \mid\left(G^{2 g} \times \Theta\right)$ is a submersion. Henceforth we shall assume that $\mathcal{M}_{o}(\Theta)$ is smooth, or that the results stated apply to the smooth part of $\mathcal{M}_{o}(\Theta)$.

Consider any $\alpha=(h, c) \in\left(\Pi \mid G^{2 g} \times \Theta\right)^{-1}(e)$. Equations (3.5) and (3.6) imply that $X \in \operatorname{ker}\left(d \Pi_{\alpha} \mid G^{2 g} \times \Theta\right)^{*}$ if and only if $f_{j+2} X=f_{j-1} X$, for every $j \in J$, and $\operatorname{Ad}(K) X \in\left(c^{-1} T_{c} \Theta\right)^{\perp}$. The relations $f_{j+2} X=f_{j-1} X$ imply (see [KS1, Proposition IV.C] or [Go]) that $\operatorname{Ad}\left(\alpha_{l}\right) X=X$ for each $i$, and that therefore (since $c=K^{-1}$ ) 
$\operatorname{Ad}(c) X=X$. Observing that $\left(c^{-1} T_{c} \Theta\right)^{\perp}=\operatorname{ker}[\operatorname{Ad}(c)-1]$ (see Remark 3.5(i) below), we see that

$$
\operatorname{ker}\left(d \Pi_{\alpha} \mid G^{2 g} \times \Theta\right)^{*}=\operatorname{ker} d K_{h}^{*} .
$$

Now let

$$
\gamma_{\alpha}: G \rightarrow G^{2 g} \times G: x \mapsto x \alpha x^{-1}
$$

and $p r_{1}: G^{2 g} \times G \rightarrow G^{2 g}$ the projection on the first factor. Then, as we have already remarked (by [KS1: Proposition IV.C] and [Go]):

$$
\operatorname{ker}\left(p r_{1} \circ \gamma_{\alpha}\right)^{\prime}=\operatorname{ker} d K_{h}^{*}
$$

wherein both derivatives are being viewed, by appropriate left-translations, to be maps between $g$ and $g^{2 g}$. Suppose that $\Theta$ is chosen to be a conjugacy class through a regular value of $\bar{K}$. Combining the observations made above we see that then $\operatorname{ker} \gamma_{\alpha}^{\prime}=\{0\}$. Thus the isotropy groups of the action of $G$ on $\mathcal{M}_{o}(\Theta)$ are all discrete. By the theory of transformation groups there is a non-empty open subset $\mathcal{M}_{o}(\Theta)^{\prime}$ of the manifold $\mathcal{M}_{o}(\Theta)$ consisting of points of minimal isotropy, and the corresponding subset $\mathcal{M}(\Theta)^{\prime}$ of $\mathcal{M}(\Theta)$ is a smooth manifold of dimension

$$
\begin{aligned}
\operatorname{dim} \mathcal{M}(\Theta)=\operatorname{dim}\left(\mathcal{M}_{o}(\Theta)\right)-\operatorname{dim} G & =(2 g) \operatorname{dim} G+\operatorname{dim} \Theta-\operatorname{dim} G-\operatorname{dim} G \\
& =(2 g-2) \operatorname{dim} G+\operatorname{dim} \Theta .
\end{aligned}
$$

If $\Theta$ is a generic conjugacy class then $\operatorname{dim}(\Theta)=\operatorname{dim} G-\operatorname{dim} T$ for any maximal torus $T$ in $G$.

The results we shall prove concerning 2-forms apply to $\mathcal{M}(\Theta)^{\prime}$, which we shall refer to as the 'smooth part' of $\mathcal{M}(\Theta)$. The results may also be meaningful when restricted to the subsets of $\mathcal{M}(\Theta)$ corresponding to other isotropy groups, but we shall not investigate such issues in the present work.

3.3. The 2-forms $\bar{\Omega}_{1}$ and $\Lambda$. The 2-form $\Lambda$ on $G^{2 g}$ is defined as follows ( $\Lambda$ was obtained in $[\mathrm{KS} 1]$ in describing the symplectic structure of the moduli space of flat connections over compact oriented surfaces without boundary). Consider $\alpha=$ $\left(a_{1}, b_{1}, \ldots, a_{g}, b_{g}\right) \in G^{2 g}$, and vectors $H^{(l)}=\left(A_{1}^{(i)}, \ldots, B_{g}^{(t)}\right) \in \underline{g}^{2 g}$, for $i=1,2$. Then:

$$
\Lambda_{\alpha}\left(\alpha H^{(1)}, \alpha H^{(2)}\right)=\frac{1}{2} \sum_{1 \leqq i, k \leqq 4 g} \varepsilon_{i k}\left\langle f_{i-1}^{-1} H_{l}^{(1)}, f_{k-1}^{-1} H_{k}^{(2)}\right\rangle,
$$

where the $f_{l}, H_{i}^{(\cdot)}$ are defined as in Sect. 3.2, and

$$
\varepsilon_{l k}= \begin{cases}1 & \text { if } i<k \\ -1 & \text { if } i>k \\ 0 & \text { if } i=k\end{cases}
$$

The map $\Pi$ being a submersion, $\Pi^{-1}(e)=\mathcal{M}_{o}$ is a smooth submanifold of $G^{2 g+1}$. Using the projection $p r_{1}: \Pi^{-1}(e) \rightarrow G^{2 g}$, we pull back $\Lambda$ to a 2 -form $\bar{\Omega}_{1}$ on $\Pi^{-1}(e)$ :

$$
\bar{\Omega}_{1}=p r_{1}^{*} \Lambda \text {. }
$$

The 2-form $\bar{\Omega}_{1}$ was introduced and studied in [KS3], where it was denoted $\bar{\Omega}_{\text {new }}$. It is shown in [KS3, Proposition 3.3] that $\bar{\Omega}_{1}$ corresponds to the 2 -form $\Omega+\Omega_{e x}$ on $\mathcal{A}$. In the present work we are interested mainly in the restriction of $\bar{\Omega}_{1}$ to $\mathcal{M}_{o}(\Theta)$. 
3.4. The 2-form $\bar{\Omega}_{o, \Theta}$ on $\mathcal{M}_{o}(\Theta)$. Recall that $\mathcal{M}_{o}(\Theta) \subset G^{2 g} \times \Theta$; a tangent vector to $\mathcal{M}_{o}(\Theta)$ at a point $\left(a_{1}, b_{1}, \ldots, a_{g}, b_{g}, c\right)=(h, c)$ is of the form $(h H, c C)$, for some $H \in g^{2 g}$ and $C \in g\left(H\right.$ and $C$ are restricted by $d \Pi(h H, c C)=0$ and by $\left.c C \in T_{c} \Theta\right)$. It will often be convenient to write this tangent vector simply as $(H, C)$, it being understood from the context that this is a vector in $T_{(h, c)} \mathcal{M}_{o}(\Theta)$. We define the 2-form $\bar{\Omega}_{o, \Theta}$ on $\mathcal{M}_{o}(\Theta)$ by:

$$
\bar{\Omega}_{o, \Theta}=\bar{\Omega}_{1}-\frac{1}{2} p r_{\Theta}^{*} v_{\Theta}
$$

where $\operatorname{pr}_{\Theta}: \mathcal{M}_{o}(\Theta) \rightarrow \Theta$ is the projection on the second factor (recall that $\left.\mathcal{M}_{o}(\Theta) \subset G^{2 g} \times \Theta\right)$ and $v_{\Theta}$ is the 2 -form on $\Theta$ given by

$$
\left(v_{\Theta}\right)_{c}(c X, c Y)=\left\langle\tilde{X}_{c},\left[\operatorname{Ad}\left(c^{-1}\right)-\operatorname{Ad}(c)\right] \tilde{Y}_{c}\right\rangle
$$

with

$$
\begin{gathered}
X=\left[\operatorname{Ad}\left(c^{-1}\right)-1\right] \tilde{X}_{c}, \\
Y=\left[\operatorname{Ad}\left(c^{-1}\right)-1\right] \tilde{Y}_{c} .
\end{gathered}
$$

Thus, for any $c \in \Theta$, considering the map $\Phi_{c}: G \rightarrow \Theta: x \mapsto x c x^{-1}$, we have

$$
\left(\Phi_{c}^{*} v_{\Theta}\right)_{e}(X, Y)=\left\langle X,\left[\operatorname{Ad}\left(c^{-1}\right)-\operatorname{Ad}(c)\right] Y\right\rangle .
$$

It should be noted that Eq. (3.9) can be taken to specify $\bar{\Omega}_{o, \Theta}$ as a 2 -form on $G^{2 g} \times \Theta$ (this will be used in the proof of Theorem 4.1).

3.5. Remarks. (i) Since $G \mapsto \Theta: x \mapsto x c x^{-1}$ is a submersion (see Sect. 2.3), every vector in $T_{c} \Theta$ has the form $c .\left[\operatorname{Ad}\left(c^{-1}\right)-1\right] \tilde{X}$ for some $\tilde{X} \in g$. The right side of Eq. (3.10) is independent of the specific choices of $\tilde{X}_{c}, \tilde{Y}_{c}$ satisfying $(3.10 \mathrm{a}, \mathrm{b})$.

(ii) The 2-form $v_{\Theta}$ is not left-invariant, but, as shown in Lemma 3.7, it is invariant under the conjugation action of $G$ on $\Theta$.

The following result explains the significance of the 2-form $\bar{\Omega}_{o, \Theta}$, and describes the 2-form $\bar{\Omega}_{\Theta}$ on $\mathcal{M}(\Theta)$ which is our principal object of interest.

3.6. Theorem. The 2-form $\bar{\Omega}_{o, \Theta}$ is closed, gauge-invariant (i.e. invariant under the conjugation action of $G$ on $\left.\mathcal{M}_{o}(\Theta)\right)$, and $\bar{\Omega}_{o, \Theta}(X, Y)$ vanishes when $X$ or $Y$ is tangent to the orbit of the $G$-action on $\mathcal{M}_{o}(\Theta)$. Thus it induces a closed 2-form, which we denote $\bar{\Omega}_{\Theta}$, on the smooth part of $\mathcal{M}(\Theta)=\mathcal{M}_{o}(\Theta) / G$.

The proof of Theorem 3.6 will appear at the end of this section.

We shall now present some results describing the significant features of the 2-form $v_{\Theta}$.

3.7. Lemma. Let $c \in \Theta$ and consider the map $\Phi_{c}: G \rightarrow \Theta: x \mapsto x c x^{-1}$. Then

$$
\left(\Phi_{c}^{*} v_{\Theta}\right)_{x}(x X, x Y)=\left\langle X,\left[A d\left(c^{-1}\right)-A d(c)\right] Y\right\rangle .
$$

Thus for any point $c^{\prime}=x c x^{-1} \in \Theta$ and for any $X_{c^{\prime}}, Y_{c^{\prime}} \in c^{\prime-1} T_{c^{\prime}} \Theta$ :

$$
\left(v_{\Theta}\right)_{c^{\prime}}\left(c^{\prime} X_{c^{\prime}}, c^{\prime} Y_{c^{\prime}}\right)=\left\langle X^{\prime},\left[A d\left(c^{-1}\right)-A d(c)\right] Y^{\prime}\right\rangle
$$


where $X^{\prime}=\left[A d\left(c^{-1}\right)-1\right]^{-1} A d\left(x^{-1}\right) X_{c^{\prime}}$, and $Y^{\prime}$ is related to $Y_{c^{\prime}}$ similarly, the notation $\left[A d\left(c^{-1}\right)-1\right]^{-1} Y$ signifying any element $Z$ for which $\left[A d\left(c^{-1}\right)-1\right] Z=Y$.

Remark. Note that the expression on the right side of $\left(3.11^{\prime}\right)$ is actually independent of the choice of $x$ satisfying $c^{\prime}=x c x^{-1}$.

Proof. By Remark 3.5(i) we can write

$$
\begin{aligned}
X_{c^{\prime}} & =\left[\operatorname{Ad}\left(c^{\prime-1}\right)-1\right] \tilde{X}_{c^{\prime}}=\operatorname{Ad}(x)\left[\operatorname{Ad}\left(c^{-1}\right)-1\right] \operatorname{Ad}\left(x^{-1}\right) \tilde{X}_{c^{\prime}}, \\
Y_{c^{\prime}} & =\left[\operatorname{Ad}\left(c^{-1}\right)-1\right] \tilde{Y}_{c^{\prime}}=\operatorname{Ad}(x)\left[\operatorname{Ad}\left(c^{-1}\right)-1\right] \operatorname{Ad}\left(x^{-1}\right) \tilde{Y}_{c^{\prime}} .
\end{aligned}
$$

Then the right side of Eq. $\left(3.11^{\prime}\right)$ equals

$$
\begin{aligned}
& =\left\langle\operatorname{Ad}\left(x^{-1}\right) \tilde{X}_{c^{\prime}},\left[\operatorname{Ad}\left(c^{-1}\right)-\operatorname{Ad}(c)\right] \operatorname{Ad}\left(x^{-1}\right) \tilde{Y}_{c^{\prime}}\right\rangle \\
& =\left\langle\tilde{X}_{c^{\prime}},\left[\operatorname{Ad}\left(c^{\prime-1}\right)-\operatorname{Ad}\left(c^{\prime}\right)\right] \tilde{Y}_{c^{\prime}}\right\rangle \\
& =\left(v_{\Theta}\right)_{c^{\prime}}\left(c^{\prime} X_{c^{\prime}}, c^{\prime} Y_{c^{\prime}}\right) .
\end{aligned}
$$

This proves Eq. $\left(3.11^{\prime}\right)$. Equation (3.11) is equivalent to Eq. $\left(3.11^{\prime}\right)$.

3.8. Lemma. The 2-form $v_{\Theta}$ is invariant under the conjugation action of $G$, and thus $p r_{\Theta}^{*} v_{\Theta}$ is gauge invariant, where $p r_{\Theta}: G^{2 g} \times \Theta \rightarrow \Theta$ is the projection on the second factor.

Proof. The map $\Phi_{c}: G \rightarrow \Theta: x \mapsto x c x^{-1}$ commutes with the $G$-actions (by left translation on $G$, and by conjugation on $\Theta$ ). Thus, since $\Phi_{c}^{*} v_{\Theta}$ is left-invariant, and since $\Phi_{c}$ is a surjective submersion, it follows that $v_{\Theta}$ is invariant under conjugation. The argument may be formulated more explicitly as follows. Conjugation by $x$ carries $c \in \Theta$ to $c^{\prime}=x c x^{-1} \in \Theta$, and any vector $c X_{c} \in T_{c} \Theta$ to $c^{\prime} \operatorname{Ad}(x) X_{c} \in T_{c^{\prime}} \Theta$. From Lemma 3.7, we then obtain

$$
\begin{aligned}
\left(v_{\Theta}\right)_{c^{\prime}}\left(c^{\prime} \operatorname{Ad}(x) X_{c}, c^{\prime} \operatorname{Ad}(x) Y_{c}\right) & =\left\langle\rho_{c} X_{c},\left[\operatorname{Ad}\left(c^{-1}\right)-\operatorname{Ad}(c)\right] \rho_{c} Y_{c}\right\rangle \\
& =\left.\left(v_{\Theta}\right)\right|_{c}\left(c X_{c}, c Y_{c}\right),
\end{aligned}
$$

where $\rho_{c}=\left[\operatorname{Ad}\left(c^{-1}\right)-1\right]^{-1}$, thus showing that $v_{\Theta}$ is conjugation-invariant.

3.9. Proposition. The 2-form $v_{\Theta}$ is smooth and

$$
d v_{\Theta}=-\left.\lambda\right|_{\Theta},
$$

where $\lambda$ is the left-invariant 3-form on $G$ specified on $T_{e} G$ by:

$$
\lambda(A, B, C)=\langle[A, B], C\rangle .
$$

Proof. Fix $c \in \Theta$. Consider the map:

$$
\Phi_{c}: G \rightarrow \Theta: x \mapsto x c x^{-1} .
$$

Recall Eq. (3.11):

$$
\left(\Phi_{c}^{*} v_{\Theta}\right)_{p}(p X, p Y)=\left\langle X,\left[\operatorname{Ad}\left(c^{-1}\right)-\operatorname{Ad}(c)\right] Y\right\rangle,
$$


where $X, Y \in \underline{g}$. Since $\Phi_{c}^{*} v_{\Theta}$ is a smooth left-invariant 2 -form on $G$, its derivative is calculated as follows:

$$
d\left(\Phi_{c}^{*} v_{\Theta}\right)(X, Y, Z)=-\Phi_{c}^{*} v_{\Theta}([X, Y], Z)-\Phi_{c}^{*} v_{\Theta}([Z, X], Y)-\Phi_{c}^{*} v_{\Theta}([Y, Z], X) .
$$
have:

Furthermore, by computing $d \Phi_{c}$ and from the definition of $\lambda$ in Eq. (3.13), we

$$
\Phi_{c}^{*} \lambda(X, Y, Z)=\left\langle\left[\left(\operatorname{Ad}\left(c^{-1}\right)-1\right) X,\left(\operatorname{Ad}\left(c^{-1}\right)-1\right) Y\right],\left(\operatorname{Ad}\left(c^{-1}\right)-1\right) Z\right\rangle .
$$

It is readily verified by computation (using Ad-invariance of the inner-product $\langle\cdot, \cdot\rangle)$ that Eqs. (3.14a), (3.14) and (3.15) imply

$$
d\left(\Phi_{c}^{*} v_{\Theta}\right)=-\Phi_{c}^{*} \lambda .
$$

It follows from Eq. (3.14) that $\Phi_{c}^{*} v_{\Theta}$ is : (i) smooth, (ii) invariant under right translations by elements of $Z(c)$, and (iii) vanishes on $(p X, p Y)$ if $p X$ or $p Y$ is in $T_{p}(p . Z(c))$. Since the quotient map $p_{c}: G \rightarrow G / Z(c)$ is a submersion, there is a 2-form $v_{\Theta}^{c}$ on $G / Z(c)$ such that

$$
\Phi_{c}^{*} v_{\Theta}=p_{c}^{*} v_{\Theta}^{c}
$$

Being a submersion, $p_{c}$ has smooth local sections. Applying $s^{*}$, where $s$ is one such smooth local section, to the preceding equation we obtain $v_{\Theta}^{c}=s^{*}\left(\Phi_{c}^{*} v_{\Theta}\right)$. Thus $v_{\Theta}^{c}$ is smooth. The map $\Phi_{c}$ induces a diffeomorphism $\bar{\Phi}_{c}: G / Z(c) \rightarrow \Theta$, i.e. $\bar{\Phi}_{c} \circ p_{c}=\Phi_{c}$. Then:

$$
p_{c}^{*} v_{\Theta}^{c}=\Phi_{c}^{*} v_{\Theta}=\left(\bar{\Phi}_{c} \circ p_{c}\right)^{*} v_{\Theta}=p_{c}^{*} \bar{\Phi}_{c}^{*} v_{\Theta}
$$

Since $p_{c}$ is a submersion, we conclude that $v_{\Theta}^{c}=\bar{\Phi}_{c}^{*} v_{\Theta}$, and therefore $v_{\Theta}=$ $\left(\bar{\Phi}_{c}^{-1}\right)^{*} v_{\Theta}^{c}$ is smooth.

From Eq. (3.16) it now follows that

$$
\Phi_{c}^{*}\left(d v_{\Theta}+\lambda\right)=0 .
$$

Since $\Phi_{c}$ is a submersion onto $\Theta$, we conclude that $d v_{\Theta}+\left.\lambda\right|_{\Theta}=0$.

3.10. Lemma. If $X, Y \in T_{\alpha} \mathcal{M}_{o}(\Theta)$, for some $\alpha \in \mathcal{M}_{o}(\Theta)$, and if $Y$ is tangent to the $G$-orbit in $\mathcal{M}_{o}(\Theta)$ through $\alpha$, then $\bar{\Omega}_{o, \Theta}(X, Y)=0$.

Proof. From Eq. (3.9) we have:

$$
\left.\bar{\Omega}_{o, \Theta}\right|_{(h, c)}\left(\left(h H^{(1)}, c C^{(1)}\right),\left(h H^{(2)}, c C^{(2)}\right)\right)=\Lambda\left(h H^{(1)}, h H^{(2)}\right)-\frac{1}{2}\left(v_{\Theta}\right)_{c}\left(c C^{(1)}, c C^{(2)}\right)
$$

Fix a point $\alpha=(h, c)=\left(a_{1}, b_{1}, \ldots, a_{g}, b_{g}, c\right) \in \mathcal{M}_{o}(\Theta)$, and let

$$
K=b_{g}^{-1} a_{g}^{-1} b_{g} a_{g} \cdots b_{1}^{-1} a_{1}^{-1} b_{1} a_{1},
$$

viewed also as a map $G^{2 g} \rightarrow G$. The conjugation action of $G$ is described by the map $\gamma_{\alpha}: G \rightarrow \mathcal{M}_{o}(\Theta): x \mapsto\left(x a_{1} x^{-1}, \ldots, x b_{y}^{-1}, x c x^{-1}\right)$. Let $\left(h H^{(1)}, c C^{(1)}\right) \in T_{\alpha} \mathcal{M}_{o}(\Theta)$. Let $p r_{1}: G^{2 g} \times \Theta \rightarrow G^{2 g}$ be the projection on $G^{2 g}$. Then calculation using 
Eq. (3.7) shows that ( for a detailed argument see Lemma IV.A in [KS1]):

$$
\Lambda\left(h H^{(1)},\left(p r_{1} \circ \gamma_{\alpha}\right)^{\prime} X\right)=-\frac{1}{2}\left\langle\left[1+\operatorname{Ad}\left(K^{-1}\right)\right] C^{(1)}, X\right\rangle,
$$

where $C^{(1)}$ is related to $H^{(1)}$ by the equation (see Eq. (3.5)):

$$
K^{-1} d K_{h}\left(h H^{(1)}\right)+\operatorname{Ad}\left(K^{-1}\right) C^{(1)}=0 .
$$

(For Eq. (3.19) see Lemma IVA in [KS1]; note that, in comparison with the calculations in [KS1], we now must retain the term $f_{4 g}$ in the calculation at every stage - this factor was replaced by 1 in the proof in [KS1] since in that context $K$ was the identity element.)

Furthermore, if we write

$$
C^{(1)}=\left[\operatorname{Ad}\left(c^{-1}\right)-1\right] \tilde{C}^{(1)},
$$

then a simple calculation based on equation (3.19), and using $c K=e$, shows that:

$$
\Lambda\left(h H^{(1)},\left(p r_{1} \circ \gamma_{\alpha}\right)^{\prime} X\right)=\frac{1}{2}\left\langle\tilde{C}^{(1)},\left[\operatorname{Ad}\left(c^{-1}\right)-\operatorname{Ad}(c)\right] X\right\rangle,
$$

Observing that the last component of $\gamma_{\alpha}^{\prime} X$ is $\left(\gamma_{\alpha}^{\prime} X\right)_{2 g+1}=\left[\operatorname{Ad}\left(c^{-1}\right)-1\right] X$, we calculate from Eq. (3.10) and Eq. (3.21) that

$$
\Lambda\left(h H^{(1)},\left(p r_{1} \circ \gamma_{\alpha}\right)^{\prime} X\right)-\frac{1}{2}\left(v_{\Theta}\right)_{c}\left(c C^{(1)}, c\left(\gamma_{\alpha}^{\prime} X\right)_{2 g+1}\right)=0 \text {. }
$$

Proof of Theorem 3.6. Recall $\mathcal{M}_{o}(\Theta) \subset G^{2 g} \times \Theta$. Let $p r_{1}: \mathcal{M}_{o}(\Theta) \rightarrow G^{2 g}$ and $\operatorname{pr}_{\Theta}: \mathcal{M}_{o}(\Theta) \rightarrow \Theta$ be the projections on the two components. As proven in [KS3], the 2-form $\Lambda$, and hence the 2-form $\operatorname{pr}_{1}^{*} \Lambda$ on $\mathcal{M}_{o}(\Theta)$, is gauge invariant. Combined with the gauge invariance of $p r_{\Theta}^{*} v_{\Theta}$ (Lemma 3.8), this proves that $\bar{\Omega}_{o, \Theta}$ is gauge invariant. Furthermore, as was proved in [KS3, Proposition 4.6],

$$
d\left(p r_{1}^{*} \Lambda\right)=-\frac{1}{2} p r_{\Theta}^{*} \lambda,
$$

Combined with the result of Proposition 3.9, this implies

$$
d \bar{\Omega}_{o, \Theta}=d\left(p r_{1}^{*} \Lambda-\frac{1}{2} p r_{\Theta}^{*} v_{\Theta}\right)=-\frac{1}{2} p r_{\Theta}^{*} \lambda-\frac{1}{2}\left(-p r_{\Theta}^{*} \lambda\right)=0 .
$$

Combining these results with Lemma 3.10 , we deduce that $\bar{\Omega}_{o, \Theta}$ induces a closed 2-form on $\mathcal{M}(\Theta)$.

3.11. An alternative description of $\mathcal{M}(\Theta)$ and $\bar{\Omega}_{\Theta}$. The moduli space $\mathcal{M}(\Theta) \simeq$ $\mathcal{A}^{f l}(\Theta) / \mathcal{G}$ can be identified with a quotient of a subset of $G^{2 g}$ in the following way. Fix $c \in \Theta$, and recall the product-commutator $K: G^{2 g} \rightarrow G:\left(a_{1}, b_{1}, \ldots, a_{g}, b_{g}\right) \mapsto$ $b_{g}^{-1} a_{g}^{-1} b_{g} a_{g} \cdots b_{1}^{-1} a_{1}^{-1} b_{1} a_{1}$. Then the map $f: K^{-1}\left(c^{-1}\right) \rightarrow G^{2 g} \times \Theta: h \mapsto(h, c)$ induces a bijection

$$
\bar{f}: K^{-1}\left(c^{-1}\right) / Z(c) \mapsto \mathcal{M}(\Theta),
$$


where $Z(c)=\left\{x \in G: x c x^{-1}=x\right\}$. Since $f$ is continuous, so is $\bar{f}$; thus $\bar{f}$ is a homeomorphism, being a continuous bijection between compact Hausdorff spaces. Moreover, since $f$ is a smooth immersion, the map $\bar{f}$ is a diffeomorphism on appropriate smooth subsets. Pulling back $\bar{\Omega}_{\Theta}$ to $K^{-1}\left(c^{-1}\right) / Z(c)$, we obtain the 2 form $\bar{\Omega}_{\Theta}^{\prime} \stackrel{\text { def }}{=} \bar{f}^{*} \bar{\Omega}_{\Theta}$. From Eq. (3.18) we see that $\bar{\Omega}_{\Theta}^{\prime}$ is simply the 2 -form induced on $K^{-1}\left(c^{-1}\right) / Z(c)$ by the 2 -form $\Lambda$ on $K^{-1}\left(c^{-1}\right)$.

\section{Non-Degeneracy of $\overline{\boldsymbol{\Omega}}_{\Theta}$}

We approach the question of non-degeneracy of $\bar{\Omega}_{\Theta}$ using some of the ideas and results from [KS2]. We prove that $\bar{\Omega}_{\Theta}$ is non-degenerate for all generic conjugacy classes sufficiently close to, but distinct from, the identity in $G$ (the degenerate case $\Theta=\{e\}$ is essentially covered by the results in [KS2]).

4.1. Theorem. There is a non-empty open set $U \subset G \backslash\{e\}$ such that the 2-form $\bar{\Omega}_{\Theta}$ on the smooth part of $\mathcal{M}(\Theta)$ is non-degenerate for every conjugacy class $\Theta$ containing a point of $U$.

Proof. Consider the 2-form $\bar{\Omega}_{o, \Theta}$ at a point $\alpha \in \Pi^{-1}(e) \subset G^{2 g} \times \Theta$, where $\Pi$ : $G^{2 g} \times \Theta \rightarrow G:\left(a_{1}, b_{1}, \ldots, a_{g}, b_{g}, c\right) \mapsto c b_{g}^{-1} a_{g}^{-1} b_{g} a_{g} \ldots b_{1}^{-1} a_{1}^{-1} b_{1} a_{1}$. We assume that $\Theta$ is such that $\mathcal{M}_{o}(\Theta)$ is smooth (as in Sect. 3.2).

Consider the orbit map of the $G$ action on $G^{2 g}: \gamma_{\alpha}: G \rightarrow G^{2 g}: x \mapsto x \alpha x^{-1}$. Since $\Pi \circ \gamma_{\alpha}(G)=\{e\}$, we have $d \Pi_{\alpha} \circ \gamma_{\alpha}^{\prime}=0$, and so $d \Pi_{\alpha}^{*}(\underline{g}) \perp \gamma_{\alpha}^{\prime}(\underline{g})$. Here $d \Pi_{\alpha}$ and $\gamma_{\alpha}^{\prime}$ are being taken as maps between $\underline{g}$ and $\underline{g}^{2 g}$, by means of appropriate left translations. We write the tangent space $T_{\chi}\left(G^{2 g} \times \Theta\right)$ as an orthogonal sum:

$$
T_{\alpha}\left(G^{2 g} \times \Theta\right)=d \Pi_{\alpha}^{*}(\underline{g}) \oplus \gamma_{\alpha}^{\prime}(\underline{g}) \oplus T_{\bar{\alpha}} \mathcal{M}(\Theta) .
$$

Here we have written $\Pi$ for $\Pi \mid\left(G^{2 g} \times \Theta\right)$, and the third factor is the orthogonal complement of $d \Pi_{\alpha}^{*}(g) \oplus \gamma_{\alpha}^{\prime}(g)$, and can be identified with the tangent space to $\mathcal{M}(\Theta)$ at $\bar{\alpha}$, where $\bar{\alpha} \in \mathcal{M}(\Theta)$ corresponds to $\alpha \in \mathcal{M}_{o}(\Theta)$.

Recall from the note at the end of Sect. 3.4 that $\bar{\Omega}_{o, \Theta}$ is meaningful as a 2-form on $G^{2 g} \times \Theta$. This 2-form $\bar{\Omega}_{o, \Theta}$ may be viewed as a linear map on $\alpha^{-1} T_{\alpha}\left(G^{2 g} \times \Theta\right)$ by writing $\left\langle X, \bar{\Omega}_{o, \Theta} Y\right\rangle$ for $\bar{\Omega}_{o, \Theta}(X, Y)$. Then Lemma 3.10 implies that $\bar{\Omega}_{o, \Theta}$ maps $\gamma_{\alpha}^{\prime}(g)$ into $d \Pi_{\alpha}^{*}(\underline{g})$. Thus, we may write a matrix of $\bar{\Omega}_{o, \Theta}$ relative to an orthonormal basis, consisting of orthonormal bases in the subspaces of the decomposition in (4.1), in the $3 \times 3$ block form:

$$
\bar{\Omega}_{o, \Theta}=\left(\begin{array}{ccc}
* & Q_{\Theta} & * \\
-Q_{\Theta}^{*} & 0 & 0 \\
* & 0 & p^{*} \bar{\Omega}_{\Theta}
\end{array}\right)
$$

Here $Q_{\Theta}$ is the restriction of $\bar{\Omega}_{o, \Theta}$ to a map from $\gamma_{\alpha}^{\prime}(\underline{g})$ to $d \Pi_{\alpha}^{*}(\underline{g})$. Hence,

$$
\operatorname{det}\left(\bar{\Omega}_{o, \Theta}\right)=\operatorname{det}\left(Q_{\Theta}\right)^{2} \operatorname{det}\left(\bar{\Omega}_{\Theta}\right),
$$

where the determinants (significant only up to multiplication by \pm 1 ) are calculated using appropriate orthonormal bases. Therefore the non-degeneracy of $\bar{\Omega}_{\Theta}$ will be 
implied by the non-vanishing of $\operatorname{det}\left(\bar{\Omega}_{o, \Theta}\right)$. This determinant can alternatively be evaluated using the decomposition $T_{\alpha}\left(G^{2 g} \times \Theta\right)=T_{h} G^{2 g} \oplus T_{c} \Theta$, where $\alpha=(h, c)$, as follows; from Eqs. (3.8) and (3.9):

$$
\bar{\Omega}_{o, \Theta}=\left(\begin{array}{cc}
\Lambda_{h} & 0 \\
0 & -\frac{1}{2}\left(v_{\Theta}\right)_{c}
\end{array}\right)
$$

Hence

$$
\operatorname{det} \bar{\Omega}_{o, \Theta}=\operatorname{det} \Lambda_{h} \operatorname{det}\left(-\frac{1}{2} v_{\Theta}\right)_{c} .
$$

It has been shown in [KS2] that $\left|\operatorname{det} \Lambda_{h}\right|=1$ when $h \in K^{-1}(e)$. Since $\left(\operatorname{det} \Lambda_{h}\right)^{2}$ depends continuously on $h$, there is an open neighborhood $\tilde{U}$ of $e$ in $G$, such that $\left|\operatorname{det} \Lambda_{h}\right|>0$ whenever $h \in K^{-1}(\tilde{U})$.

As we have noted before (Remark 3.5(i)), $c^{-1} T_{c} \Theta=\left[\operatorname{Ad}\left(c^{-1}\right)-1\right](g)$. By "diagonalizing" $\operatorname{Ad}(c)$ on $g$, we obtain a block diagonal matrix for $\operatorname{Ad}(c)$, with $2 \times 2$ rotation matrices on the diagonal as follows:

$$
\operatorname{Ad}(c)=\left(\begin{array}{ccccc}
I & 0 & 0 & \ldots & 0 \\
0 & R_{\phi_{1}} & 0 & \ldots & 0 \\
0 & 0 & R_{\phi_{2}} & \ldots & 0 \\
0 & 0 & 0 & \ldots & R_{\phi_{m}}
\end{array}\right) .
$$

Here $R_{\phi}$ is the $2 \times 2$ rotation matrix

$$
R_{\phi}=\left(\begin{array}{cc}
\cos \phi & -\sin \phi \\
\sin \phi & \cos \phi
\end{array}\right) .
$$

Recall from Eqs. (3.10) and (3.10a,b) that:

$$
\left(v_{\Theta}\right)_{c}(c X, c Y)=\left\langle\rho_{c} X,\left[\operatorname{Ad}\left(c^{-1}\right)-\operatorname{Ad}(c)\right] \rho_{c} Y\right\rangle
$$

where $\rho_{c}=\left[\operatorname{Ad}\left(c^{-1}\right)-1\right]^{-1}$, these being inverses on the appropriate spaces.

In terms of the representation in Eq. (4.7), the matrix for $v_{\Theta}$ is thus again block diagonal, consisting only of the $m$ lower $2 \times 2$ blocks. From (4.8) and (4.6) it follows that each $2 \times 2$ block for $v_{\Theta}$ has the form

$$
\left(R_{\phi}-1\right)^{-1}\left(R_{\phi}^{-1}-R_{\phi}\right)\left(R_{\phi}^{-1}-1\right)^{-1}=\left(R_{\phi}+1\right)\left(R_{\phi}-1\right)^{-1} .
$$

Hence

$$
\operatorname{det} v_{\Theta}=\prod_{l=1}^{m} \frac{\operatorname{det}\left(R_{\phi_{1}}+1\right)}{\operatorname{det}\left(R_{\phi_{l}}-1\right)}=\prod_{l=1}^{m} \frac{1+\cos \phi_{i}}{1-\cos \phi_{i}} .
$$

When $\Theta$ is not a trivial conjugacy class $\{z\}$, with $z$ in the center of $G$ (this center is discrete by semisimplicity), the number $m$ is $\geqq 1$. Furthermore, when $\Theta$ is close enough to the identity, none of the $\cos \phi_{l}$ is equal to -1 . Therefore, $\operatorname{det}\left(-\frac{1}{2} v_{\Theta}\right) \neq 0$ in this case.

Combining this result with the existence of $\tilde{U}$ (see the discussion following Eq. (4.5)), we deduce the existence of an open set $U \subset G \backslash\{e\}$ for which $\operatorname{det} \vec{\Omega}_{\Theta} \neq 0$ whenever $\Theta$ contains a point in $U$. 


\section{The infinite dimensional setting}

In this section, we will identify a 2-form $\Omega_{\Theta}$ on $\mathcal{A}(\Theta)$ which induces the 2-form $\bar{\Omega}_{\Theta}$ on the finite dimensional moduli space $\mathcal{M}(\Theta)$.

As was described in Sect. 2.4, the tangent space to $\mathcal{A}$ at a point $\omega \in \mathcal{A}$ may be taken to be the space $T_{\omega} \mathcal{A}$ of all smooth $g$-valued 1-forms on $\Sigma$. If $\omega \in \mathcal{A}^{f l}(\Theta)$, we take $T_{\omega} \mathcal{A}^{f l}(\Theta)$ to be the set of all $A \in T_{\omega} A$ for which $A(m)=\left.\frac{\hat{\alpha} \omega_{\varepsilon}(m)}{\partial \varepsilon}\right|_{\varepsilon=0}$, for every $m \in \Sigma$, where $\varepsilon \mapsto \omega_{\varepsilon} \in \mathcal{A}^{f l}(\Theta)$, with $\varepsilon$ in a neighborhood of 0 , is such that $(\varepsilon, m) \rightarrow \omega_{\varepsilon}(m)$ is smooth.

Recall from Sect. 2.7 that the action of $\mathcal{G}$ on $\mathcal{A}$ is given by $(\phi, \omega) \mapsto$ $\operatorname{Ad}\left(\phi^{-1}\right) \omega+\phi^{-1} d \phi$, it follows by differentiation that the tangent space to the $\mathcal{G}$ orbit through $\omega$ may be taken to be

$$
T_{\omega}(\mathcal{G} \cdot \omega)=\{d H+[\omega, H]: \text { smooth } H: \Sigma \rightarrow \underline{g}\} .
$$

Let us also note that if $t \mapsto h_{t}(\omega)$ describes parallel transport (as described in Sect. 2.5) by $\omega$ along a curve $\kappa:[0,1] \rightarrow \Sigma$, and if $A \in T_{\omega} \mathcal{A}$, then the variation $\delta_{A} h_{t}(\omega)$ in $h_{t}(\omega)$ corresponding to the variation $A$ in $\omega$, is given by:

$$
\left.h_{t}(\omega)^{-1} \delta_{A} h_{t}(\omega) \stackrel{\text { def }}{=} h_{t}(\omega)^{-1} \frac{d}{d \varepsilon}\right|_{\varepsilon=0} h_{t}(\omega+\varepsilon A)=-\int_{0}^{t} \operatorname{Ad}\left(h_{s}^{-1}\right) A\left(\kappa^{\prime}(s)\right) d s .
$$

When $\kappa$ is the loop $C$ described in Sect. 2.1, Eq. (5.2) becomes (3.1) discussed earlier.

Recall, from Eqs. (2.2) and (3.2), the 2-forms $\Omega$ and $\Omega_{e x}$ on $\mathcal{A}$. Using the 2form $-\frac{1}{2} v_{\Theta}$, as specified through Eqs. (3.10) and (3.10a,b), as a guide, we introduce the 2 -form $\Omega_{r}$ on $\mathcal{A}(\Theta)$ by:

$$
\left.\Omega_{v}\right|_{\omega}(A, B)=-\frac{1}{2}\left\langle\alpha(1),(\operatorname{Ad}(c)-1)^{-1} \beta(1)\right\rangle+\frac{1}{2}\left\langle\beta(1),(\operatorname{Ad}(c)-1)^{-1} \alpha(1)\right\rangle,
$$

where $c=h(C ; \omega), C$ being the loop described in Sect. 2.1, and $\alpha, \beta$ are defined as in Sect. 3.1. The terms involving $(\operatorname{Ad}(c)-1)^{-1}$ are meaningful because $\alpha(1)$ and $\beta(1)$ represent variations of the holonomy $h(C ; \omega)$ when $\omega$ is varied in $\mathcal{A}(\Theta)$ (see Remark 3.5 and remarks in Sect. 3.1 in this context). More generally, by setting $(\operatorname{Ad}(c)-1)^{-1}$ to be 0 on $\operatorname{ker}[\operatorname{Ad}(c)-1]=[(\operatorname{Ad}(c)-1)(g)]^{\perp}$, we consider $\Omega_{v}$ as a 2 -form on $\mathcal{A}$. In view of Eqs. (3.9) and the remarks in Sect. 3.3, we set:

$$
\Omega_{\Theta}=\Omega+\Omega_{e x}+\Omega_{v}
$$

as a 2-form on $\mathcal{A}(\Theta)$ : more precisely, the right side of Eq. (5.4) is a 2-form on $\mathcal{A}$, whose restriction to $\mathcal{A}(\Theta)$ is $\Omega_{\Theta}$.

5.1. Proposition. Let $A, B \in T_{(\sigma)} \mathcal{A}^{f l}(\Theta)$, where $\omega \in \mathcal{A}^{f l}(\Theta)$, and suppose that $A \in$ $T_{\omega}(\mathcal{G} \cdot \omega)$; then

$$
\Omega_{\Theta}(A, B)=0 .
$$

Proof. Since $A \in T_{\omega}(\mathcal{G} \cdot \omega)$, Eq. (5.1) shows that $A=d H+[\omega, H]$ for some smooth $H: \Sigma \rightarrow \underline{g}$. A calculation carried out in [KS3, proof of Proposition 3.3] shows that

$$
\left(\Omega+\Omega_{e x}\right)(d H+[\omega, H], B)=-\frac{1}{2}\left\langle\left(1+\mathrm{Ad}^{-1}\right) H(0), \beta(1)\right\rangle,
$$


It is also shown in $[\mathrm{KS} 3$, Eq. (3.6)] that

$$
\alpha(1)=\left(1-\operatorname{Ad} c^{-1}\right) H(0) .
$$

Using this in Eq. (5.3) we have:

$$
\begin{aligned}
\Omega_{v}(d H+[\omega, H], B)= & -\frac{1}{2}\left\langle\left(1-\operatorname{Ad} c^{-1}\right) H(0),(\operatorname{Ad} c-1)^{-1} \beta(1)\right\rangle \\
& +\frac{1}{2}\left\langle\beta(1),(\operatorname{Ad} c-1)^{-1}\left(1-\operatorname{Ad} c^{-1}\right) H(0)\right\rangle \\
= & \frac{1}{2}\langle H(0), \beta(1)\rangle+\frac{1}{2}\left\langle\beta(1),\left(\operatorname{Ad} c^{-1}\right) H(0)\right\rangle \\
= & \frac{1}{2}\left\langle\left(\operatorname{Ad} c^{-1}+1\right) H(0), \beta(1)\right\rangle .
\end{aligned}
$$

Then from Eqs. (5.5a,b) and the definition of $\Omega_{\Theta}$ in Eq. (5.4), we obtain:

$$
\Omega_{\Theta}(d H+[\omega, H], B)=0 .
$$

One would like to verify the informal relation $d \Omega_{\Theta}=0$, at least on $\mathcal{A}^{f l}(\Theta)$. However, unlike the situation on $\mathcal{A}$, we cannot work with constant vector fields on $\mathcal{A}^{f l}(\Theta)$. This would necessitate computation of the Lie bracket of two non-constant vector fields on $\mathcal{A}$. The appropriate notion of Lie bracket here appears to be

$$
[A, B]_{\omega}=\left(\delta_{A} B\right)_{\omega}-\left(\delta_{B} A\right)_{\omega},
$$

where the $\delta$ 's are directional derivatives. This observation is corroborated by a calculation showing that

$$
\delta_{[A, B]} h_{s}=\delta_{A} \delta_{B} h_{s}-\delta_{B} \delta_{A} h_{s},
$$

where $s \mapsto h_{s}$ describes parallel transport along any curve on $\Sigma$.

The 2-forms $\Omega_{\Theta}$ and $\bar{\Omega}_{\Theta}$ : The 2-forms $\Omega, \Omega_{e x}$, and $\Omega_{v}$ are readily verified to be gauge invariant, and so $\Omega_{\Theta}$ is gauge invariant. Combining this with Proposition 5.1, we see that $\Omega_{\Theta}$ corresponds to a 2 -form on $\mathcal{A}^{f l}(\Theta) / \mathcal{G} \simeq \mathcal{M}(\Theta)$. The definition of $\Omega_{\Theta}$ shows that the corresponding 2-form on $\mathcal{M}(\Theta)$ is $\bar{\Omega}_{\Theta}$. This provides an understanding of $\bar{\Omega}_{\Theta}$ at the level of the infinite-dimensional space $\mathcal{A}^{f l}(\Theta)$.

Acknowledgements. A. Sengupta was supported in part by NSF Grant DMS 9400961, and in part by U.S. Army Research Office \#DAAH04-94-G-0249.

\section{References}

[A] Atiyah, M.: The Geometry and Physics of Knots. Cambridge: Cambridge University Press 1990

[AB] Atiyah, M., Bott, R.: The Yang-Mills Equations over Riemann Surfaces. Phil. Trans. R. Soc. Lond. A 308, 523-615 (1982)

[BG] Biswas, I., Guruprasad, K.: Principal bundles on open surfaces and invariant functions on Lie groups. Internat. J. Math. 4, 535-544 (1993)

[Go] Goldman, W.: The Symplectic Nature of Fundamental Groups of Surfaces. Adv. Math. 54, 200-225 (1984) 
[Hi] Hitchin, N.: The Symplectic Geometry of Moduli Spaces of Connections and Geometric Quantization. Prog. Theor. Phys. Supplement 102, 159-174 (1990)

[Hu] Huebschmann, J.: Symplectic and Poisson structures of certain moduli spaces. Preprint (1993)

[HJ] Huebschmann, J., Jeffrey, L.C.: Group Cohomology construction of symplectic forms on certain moduli spaces. International Mathematics Research Notes 6, 245-249 (1994)

[J] Jeffrey, L.C.: Extended moduli spaces of flat connections on Riemann surfaces. Math. Annalen 298, 667-692 (1994)

[JW1] Jeffrey, L., Weitsman, J.: Torus actions, moment maps, and the symplectic geometry of the moduli space of flat connections on a two-manifold. In: Low-Dimensional Topology and Quantum Field Theory, H. Osborn, ed. NATO ASI Series B315, New York Plenum Press, (1993)

[JW2] Jeffrey, L., Weitsman, J.: Toric structures on the moduli space of flat connections on a Riemann surface: Volumes and the moment map. Advances in Mathematics 109, 151-168 (1994)

[Ka] Karshon, Y.: An algebraic proof for the symplectic structure of moduli space. Proc. Amer. Math. Soc. 116, 591-605 (1992)

[KS1] King, C., Sengupta, A.: An Explicit Description of the Symplectic Structure of Moduli Spaces of Flat Connections. J. Math. Phys. Special Issue on Topology and Physics, 35, 5338-5353 (1994)

[KS2] King, C., Sengupta, A.: The Semiclassical Limit of the Two Dimensional Quantum YangMills Model. J. Math. Phys. Special Issue on Topology and Physics, 10, 5354-5361 (1994)

[KS3] King, C., Sengupta, A.: A new 2-form for connections on surfaces with boundary. Lett. Math. Phys. 34, 135-147 (1995)

[Se1] Sengupta, A.: Yang-Mills Minima over Compact Surfaces. Preprint.

[Se2] Sengupta, A.: The Semiclassical Limit for $S U(2)$ and $S O(3)$ Gauge Theory on the Torus. Commun. Math. Phys. 169, 297-314 (1995)

[We] Weinstein, A.: The symplectic structure on moduli space. In: The Andreas Floer Memorial Volume, Progress in Mathematics, Birkhauser (1994)

[Wil] Witten, E.: On Quantum Gauge Theories in Two Dimensions. Commun. Math. Phys. 141, 153-209 (1991)

[Wi2] Witten, E.: Two Dimensional Quantum Gauge Theory revisited. J. Geom. Phys. 9, 303-368 (1992)

Communicated by R.H. Dijkgraat 
